\title{
SELVA OSCURA O EN QUE ACABA LO MODERNO
}

\author{
Roberto FERNÁNDEZ \\ CAEAU. Universidad Abierta Interamericana \\ ASTRAGALO, 20 (2015) \\ Attribution-NonCommercial-ShareAlike - CC BY-NC-SA \\ Introducción al número, ISSN 2469-0503 \\ https://dx.doi.org/10.12795/astragalo.2015.i20.01
}

El momento tardomoderno podría visualizarse doblemente como un estado de consumación mercantil de los propósitos y resultados del programa vanguardista moderno y por tanto prólogo o transición a la posmodernidad cínica pero también como un efímero alcance de una última formulación utópica -la encarnada en el ideario del Team $X$ - que escoge paradójicamente como estrategia, destronar las megalomanías vanguardistas modernas pero apelando estrictamente a lo más genuino o anticonservador (diríamos además: antihistoricista) que había instaurado la novedad moderna en tanto innovación de lenguaje.

El Team X-último objeto de conocimiento posible en la historización del movimiento moderno, al cual se propone liquidar- postula de tal forma un balance selectivo del potencial socialmente crítico del lenguaje moderno para tratar de deslindarlo del proyecto universalis- ta y antirrelativista de los gurúes del diseño mundial que como Le Corbusier, Gropius o Mies parecían encantados con una internacionalización de sus propuestas sin (querer) ver que ya eran meras materias primas de procesos de generalización de culturas proyectuales del capitalismo avanzado y sus maniobras de expansión del potencial de las mercancías.

Dicho esto asumiendo cierta ingenuidad política en tales protagonistas, hecho en todo caso, de necesaria demostración como finalidad de trabajos historiográficos todavía por emprender. Lo cierto es que algunos personajes sesentistas parecieron, cerca o dentro del movimiento Team $X$, mucho más comprometidos y esclarecidos políticamente hablando, como fue el caso de Van Eyck, Price o Erskine.

Podría pensarse en la necesidad de dos series de estudios sobre la modernidad, si se quiere, el conjunto más significativo de hechos 
y circunstancias que ésta ha presentado entre 1850 y 1960 (período que abarca el corpus más habitual de la historiografía de la arquitectura moderna) pero tratando de trabajar sobre un par de nociones teóricas casi contrapuestas -canon y vanguardia- que se superponen a las más modernamente ortodoxas de utopías sociales y cultura técnica ${ }^{1}$ - como un relato diverso de tramos historizados o periodizaciones geo-temporales para introducir la perspectiva de ejes teóricos que tienen cada uno su propio proceso y por tanto, su propio devenir histórico.

Ambas series de estudios se articularían así sobre ejes temáticos diversos. En la primera serie prevalece la idea general del surgimiento y consolidación de la ciudad como espacio de modernización socio-técnica: la ciudad, básicamente industrial, se complejiza social y tecnológicamente de manera vertiginosa y sobre dicho proceso se motorizan fuerzas ideológicas que pretenden alcanzar alguna clase de mejoramiento social en la línea prevaleciente de un pensamiento socialista que trata de aplicar las ideas abstractas del iluminismo orientada al desarrollo de una sociedad más igualitaria articulada en la acción de un concepto de Estado benefactor fuertemente protagónico ya desde Bismarck.

Esas fuerzas instalan una idea de diseño total -o de fe extrema en la productividad proyectual- que expande la arquitectura tradicional hacia la dimensión urbana entendida como un mega-artefacto diseñable y hacia la dimensión objetual del paisaje cotidiano material y simbólico, programa que encontrará diferentes sistematizaciones ideológico-didácticas, desde el modelo del Beaux Arts hasta las guilds y workshops británicos, el Wiener Werkstatten, el Artel checo, el Werkbund, el Vkhutemas o el
Bauhaus, experiencias todas que caducan sobre los 30 con la llegada de expresiones totalitarias y el viento que llevará a la II Guerra Mundial.

Es cierto que ese leit motiv moderno de expandir el control proyectual de la nueva ciudad industrial y su pasaje a sociedad metropolitana tropieza gravemente -notoriamente en el ideario de William Morris- con la incapacidad de articular fecundamente arte o diseño e industria y esa incapacidad recae en la utopía de garantizar una ética del diseño basada en la artesanía ignorando no sólo el modo de producción industrial sino más aún, el estatuto de mercancía de cualquier objeto moderno. Aunque cabría todavía dudar de la ingenuidad política baushausiana si coincidimos con $\mathrm{Hal}$ Foster cuando menciona que Jean Baudrillard adjudica a esa escuela el mérito del pasaje de una economía política del producto a una economía política del signo.

El intento de salvaguarda aurática de las cosas del diseño -frente al imperativo de una sociedad industrial y comercial en frenética expansión- condena al modernismo a protagonismos bien relativos dentro de la vida nerviosa de las ciudades y limita la actividad de los proyectistas a niveles selectos del cuadro del consumo social.

Tanto las causas como los resultados de la segunda guerra -diríamos, el estatuto neocapitalista de Bretton Woods y la afirmación de un orden geopolítico internacional bipolar según surge de los acuerdos de Yalta- plantean algunos corolarios que modelarían la historia subsiguiente tales como el tránsito hacia formas más sutiles aunque más brutales de capitalismo, el debilitamiento de las formas del Estado (y dentro de ello, directamente la caída de los Estados socialistas) y la irrupción de la esfera compleja 
del Mercado y consecuentemente, cambios radicales en la producción y el consumo y por tanto nuevas condiciones culturales.

En ese esquema el pensamiento proyectual y la institución arquitectónica se escinden entre inserción en el Mercado y profundización de una utopía crítica negativa, todo ello frente a la desaparición del Estado como promotor de arquitectura entendida como prestación social.

El primer grupo de estudios al que aludimos refiere así más bien a un proceso de evolución positiva (y positivista) del arranque de las relaciones entre los fenómenos sociales de la modernización y los epifenómenos culturales de la modernidad: se advertiría un momento formativo y optimista anclado en la teoría general de cierto pensamiento utópico que funge como intento de articulación entre modernización y modernidad y en el programa de organizar unas prácticas proyectuales que bajo la tutela genérica de pensar y construir la ciudad van a manifestarse lingüísticamente alrededor del procesamiento de las novedades tecnológicas dando curso a una suerte de dimensión estética o artística basada en lo técnico.

El peso que lo técnico adquirirá en reorganizar todas las prácticas proyectuales es un importante síntoma para hacer entrar en crisis el procedimiento Beaux-Arts, según el cual lo técnico era meramente una dimensión fáctica muy ulterior y subsidiaria del trabajo teórico de la composición. Las cosas debían pensarse en una dimensión abstracta y canónica y su materialización resultaría la mera consecuencia de aprovecharse de disponibilidades técnicas que no requerían ser objeto de reflexión proyectual. En ese orden, los intereses técnicoexpresivos de proyectistas académicos como sería el caso de Soufflot, son francamente ex- cepcionales y de tal forma, el recentramiento de lo técnico (como había ocurrido por caso, en el medioevo) propio de la modernidad es un atributo explícito de su entidad y advenimiento histórico como nueva fase de pensamiento y praxis proyectuales.

La importancia de lo técnico ya no como fase instrumental sino como plataforma definidora de nuevos estatutos culturales será correlativa de la declinación de los sistemas discursivos que solían espesar la significación de los proyectos: sin embargo los estudios que situamos en esa primera serie no van a manifestar la desaparición de los sistemas ornamentales pero si su paulatina puesta en crisis sobre todo a partir de caracterizar dichos sistemas comunicativos como agregados o suplementos a la cuestión principal de unos proyectos producidos en sintonía con las novedades tecnológicas.

Esta suplementariedad o subalternidad de lo ornamental (pero que no significa en todo caso una reducción de la potencia de sus discursos y metadiscursos) será por caso ejemplar en Otto Wägner y será asimismo claramente advertida tal tensión en los planteos de Adolf Loos, que sin embargo fuera de sus anticipaciones teóricas, no logra resolverla plenamente en sus propios trabajos.

El eje de la segunda serie se liga más a la asunción del fracaso del ideario iluminista y a la constatación que el progresismo sociourbano es inviable y que los procesos tecnológicos quedarán en su lógica expansiva, fuera de la pretensión del control de los proyectistas. Arrancaría de tal suerte una especie de fase moderna negativa, que ya parece dar cuenta en sus protagonistas del cese de la ilusión propia de un proyecto total (utópico) capaz a la vez de modelar (técnicamente) ciudad y sociedad. 
Este fracaso en el intento de controlar la producción técnica de los objetos se evidenciará ejemplarmente en Le Corbusier y es lo que me parece que inspiró a Siegfrid Giedeon a escribir con poco menos de media década de diferencia, sus dos historias ${ }^{2}$.

El deseo de Corbusier de hacerse cargo de la cultura técnica del proyecto está evidenciado en los estudios de Beatriz Colominas ${ }^{3}$ cuando investiga en la época en el maestro suizo editaba la revista Esprit Nouveau, en la que se ocupaba no sólo de escribir y encargar escritos sino también en conseguir publicidad, lo que lo llevaba a visitar a muchos industriales de materiales y dispositivos de la construcción, temas con los cuáles además, organizaba vastos archivos documentales de folletos y propagandas.

Es así que emerge hacia los 30 -pero como veremos, recogiendo una idea pesimista más pertinaz en el tiempo- la conciencia de una ciudad desgarrada, fragmentaria y que ya no podrá garantizar coherencia (y coexistencia) social ni física: de la que su imagen ideotécnica mas fuerte -el plan- entendida como correlato estricto entre actividad social y espacio urbano pierde progresivamente sentido.

Creo que este segundo eje de análisis explica el concepto de vanguardia, tanto como emergencia exasperada del control de autor, como asunción del carácter inevitablemente disruptivo de la arquitectura respecto de la ciudad y como reinserción de la arquitectura en las características teóricas y dispositivas del arte conceptual moderno.

La oscilación y articulación teórica entre canon y vanguardia parece plantear, a la manera hegeliana, un fermento pleno de contradicción, pero justamente esa interdependencia-que suele incluir un factor de negación o reversión- aparece como un motor de modernidad y una dialéctica que nutre procesos históricos: los aportes vanguardistas (que suelen emerger como rupturistas) estabilizándose en cierta norma política y culturalmente instaurada -el canon- y nuevos cuestionamientos y apartamientos de tal norma desplegando nuevas acciones vanguardistas.

Hay muchos casos de vanguardistas aurorales o pro-canónicos -como Bernini, Boullée o Le Corbusier- y otros tantos de vanguardistas crepusculares o pos-canónicos -como Piranesi, Soane o Gaudí: los primeros suelen ser luminosos y esperanzados, abiertos a ofrecer términos para que sus aportes vanguardistas fructifiquen en sistemas canónicos futuros y piensan y actúan así para adelante; los segundos resultan más bien oscuros y apocalípticos, abocados a un trabajo disolutorio y crítico sobre los materiales precedentes.

Veremos, siguiendo a Bürger ${ }^{4}$, como por una parte la noción de vanguardia resulta una institución esencialmente moderna (y aquí entonces, para ciertos personajes, está trasladada en el tiempo; antedatada) y por otra parte, la idea misma de vanguardia parece metodológica y productivamente ser bien diferente de la acción del Movimiento Moderno que en todo caso sería otra institución o más bien, momento evolutivo en el devenir histórico de las Artes.

Asimismo el adjetivo moderno debe ser entendido doblemente como una referencia histórica que alude a un momento de tal devenir y que se constituye así en una forma o modo de constituir un sistema artístico de prácticas y productos que emergería a fines del siglo XIX, pero también como una alusión más cualitativa, intemporal o extra-histórica que remite en cualquier época a un modo de nombrar los fe- 
nómenos que intentan cuestionar o superar lo que viene dado por la tradición o el canon: esa es la manera que le atribuye Claude Perrault en 1692 cuando presenta la querelle entre antiguos y modernos o el concepto que instala Pietro Bellori en el siglo XVII cuando quiere adjetivar la novedad anticlasicista del pensamiento barroco, una novedad moderna que empero se constituye mediante una forma de reelaborar el material antiguo de la clasicidad, con lo cual esa idea de moderno no refiere a una innovación de lenguaje sino más bien a una innovación de procedimiento.

Dicho sea de paso, la idea de postproducción que presenta el crítico de arte Nicolas Bourriaud ${ }^{5}$ vuelve a instalar esa idea reelaborativa como base explicativa central del presente estético de inicios del siglo XXI, en este caso tomándose el repertorio formal moderno como materialidad a manipular.

Con lo cual, quizá con cierta resonancia de la noción nietzcheana-eliadeana de eterno retorno, parecería ratificarse una reiteración del acuñamiento y uso en varios y diferentes momentos históricos, del concepto de modernidad esencialmente entendido como novedad reelaborativa de materiales antiguos y por ello, no tanto una innovación conceptual sino más bien, instrumental.

La primera serie de estudios situadas cronológicamente en el arco que va de mitad del siglo XIX a la tercera década del XX podría entenderse como una suerte de arqueología de la modernidad canónica (eso que la historiografía llamó Movimiento Moderno) pero no porque se extinguieran sus aportaciones linguísticas sino porque se disolvieron, por causas extrainstitucionales, sus basamentos programáticos o conceptuales.
El trabajo de lenguaje de esas aportaciones no es estrictamente arqueológico (en tanto reducido a su época de producción y consumo) sino que muchos dispositivos comunicacionales siguieron o siguen vigentes.

Lo que constituye como arqueo-moderna a la historia analizada en tal primera serie de estudios es, lo repetimos, su imbricación alrededor de dos cuestiones que históricamente ahora parecen superadas: su empeño en trabajar en la idea de las utopías sociales (como si el Iluminismo político-filosófico necesitara una aplicación arquitectónica) y su interés en el montaje de una cultura técnica (como si fuera posible imaginar una arquitectura que acompañara y aplicará los ingenios de la revolución científico-tecnológica).

Las dos series de estudios, con sus diversas formas de periodización, encadenamiento y traslape, obedecen en general a la clásica noción de modernidad larga, proceso que para diversos autores implican extender esta noción al menos desde el Renacimiento como ocurrirá con las propuestas historiográficas de Manfredo Tafuri ${ }^{6}$, el que sin embargo tiende a caracterizar toda esa modernidad larga como una continua formulación entre consolatoria y negativista, como si siempre hubiera existido una conciencia acerca del carácter subalterno de la modernidad cultural respecto de la modernización socio-económica y por tanto una certeza entre utópica y fatalista acerca de la inviabilidad de un programa emancipador protagonizado por los intentos de una cultura moderna orientada a consolidar políticamente el programa iluminista: dicha noción de modernidad larga contiene así la autoafirmación de un estado de crisis.

Otros autores, como Stephen Toulmin ${ }^{7}$ también presentan la hipótesis de una moder- 
nidad larga sobre todo en la idea de los grandes cambios paradigmáticos en el pensamiento científico, filosófico y estético cuyo punto de arranque sitúa en su caso en el siglo XVII además de plantear que sólo la modernidad arquitectónica se define historiográficamente como tal circunscripta al siglo XX y a más tardar, a la última década del siglo XIX. Toulmin manifiesta cierta sorpresa de historiador de la cultura, al referirse a esa circunscripción epocal que hace de sí, la modernidad arquitectónica situándose en una realidad específica del siglo XX, lo cual pareciera implicar para este historiador, la presunción de cierta amnesia disciplinar y/o la voluntad de reducción de lo nuevo-moderno a una dimensión estrictamente frívola, en tanto consumada única o principalmente en las novedades de lenguaje.

Los sucesos que suscitan el arranque filosófico, político y científico de la modernidad responden además, para Toulmin, al origen de dos grandes tradiciones modernas: una, la canónica, racionalista o positiva, supuestamente articulada con los enunciados del método cartesiano y otra, divergente, negativa o directamente antirracionalista y relativista que podría ejemplificarse con el pensamiento de Montaigne o el de Leibnitz.

En su escrito homenaje a la desaparición de Derrida, Peter Sloterdijk ${ }^{8}$, en una serie de pequeños ensayos dedica uno a las relaciones de tal filósofo con el historiador del arte Franz Borkenau quién en su obra póstuma Fin y Principio. Sobre las generaciones de las altas culturas y el nacimiento de Occidente presenta por así decirlo un intento de periodizar como la cultura occidental se ha definido históricamente en tanto diríamos, teorías de la eternidad o complejos discursivos de encadenamiento de la praxis artística y la cosmovisión variable respecto del fenómeno de la muerte, que le motiva la noción de presentar cuatro grandes fases, a saber, la que llama egipcia (-1500/-500), lo que configuraría el momento de la antigüedad (-500/500), la denominada cristiana (500/1500) y la que caracteriza como moderna (1500-2500): cambios milenarios que van oscilando entre la pretendida negación de la muerte y búsqueda de instancias de inmortalidad o trascendencia -momentos uno y tres- y la aceptación de la mortalidad y el encauzamiento de las energías psíquicas en lo que genéricamente podríamos llamar acción política -momentos dos y cuatro: éste, de afirmarse la periodicidad milenaria, con cuatro siglos todavía por delante, de modernidad-.

Se trata si se quiere, de postular la dialéctica entre matrices culturales de trascendencia y de inmanencia -también quizá, la oscilación subjetividad/objetividad- lo cuál para Borkenau puede explicar las variaciones de discursividad que fluirían en relación a temas como la naturaleza y la técnica.

Jurgen Habermas ${ }^{9}$ también propone una idea larga de modernidad, aunque menos extensa y variada que la de Toulmin, ya que señala como origen histórico de tal noción a la aparición de los grandes textos kantianos de fines del siglo XVIII como el punto culminante de presentación de una teoría de la razón iluminista y universal, más o menos coincidente con la emergencia de las revoluciones políticas francesas y norteamericana.

Desde esa postura Habermas se vale además para presentar el proceso incompleto de la modernización como un proceso histórico de desarrollo humano y emancipación de los sujetos que no se ha consumado y por el cuál política y culturalmente vale la pena bregar todavía. 
Habermas propone otros dos argumentos complementarios que resultan atendibles: uno que la modernidad sería una formación dominantemente artístico-cultural operada como dimensión superestructural o epifenoménica de otro proceso que discurriría de modo estructural -lo que llama modernización, que en el mejor de los casos en esta etapa histórica estaría atravesando los problemas inherentes a la instauración de una figura pública de comunidad basada en el perfeccionamiento de las comunicacionesy otro que postularía la inviabilidad de una dimensión post (por ejemplo, el posmodernismo) que no podría ser entendida de manera culturalmente autónoma de una fase de postmodernización que nadie presenta como tal: este segundo argumento propondría, en línea con Marx pero quizá no con Gramsci, que no puede haber evolución o cambio autónomo de lo superestructural respecto de lo estructural.

Para Hans Robert Jauss ${ }^{10}$ la modernidad tiene como cinco momentos o umbrales de inicio: 1750 (Hume, Rousseau, Vico, Kant), 1789 (Goethe, Hegel), 1848 (Baudelaire, Benjamín), 1912 (Apollinaire) y 1967 (Calvino) que demostraría una suerte de eterno y complejo comienzo y de paso, en los análisis del último umbral -alrededor de Calvino o Borges o Pessoa- la postulación de una cierta relatividad posmoderna o lo posmoderno como momento moderno: tesis todas formuladoras de un flujo torrencial y difuso que incluso reivindicará valores para el momento posmoderno ya que Jauss, en contra de Habermas, no cree en un sojuzgamiento de la historia cultural de la modernidad a la historia social de la modernización sin que ello signifique autonomía de una respecto de la otra sino en todo caso, diversas categorías de interpretación del cambio histórico en la producción artística.
Fredric Jameson ${ }^{11}$ ofrece, dentro de una valorización del posmodernismo desde una posición new left, la idea que la modernidad y sus vestigios tardíos son derivados de la cultura recentrada en el corazón de la economía (en torno de conceptos tales como capitalismo cognitivo o tardocapitalismo) con lo cual es posible y preciso usar la historia de la modernidad como contexto explicativo del presente/futuro y así plantea argumentos como éste: Lo que ha sucedido es que en nuestros días la producción estética se ha integrado a la producción general de bienes: la frenética urgencia económica por producir nuevas líneas de productos de apariencia cada vez más novedosa (desde ropa hasta aviones) a ritmos de renovación cada vez más rápidos, le asigna ahora una función y una posición estructurales esenciales cada vez mayores a la innovación y la experimentación estéticas. Tales requerimientos económicos encuentran entonces reconocimiento en el apoyo institucional de todo tipo que resulta accesible a las nuevas formas de arte, desde las fundaciones y las donaciones hasta los museos y otras formas de mecenazgo. No obstante, de todas las artes, la arquitectura es la que, por su constitución, se encuentra más cerca de lo económico, con lo que, a través de las comisiones $y$ los valores de los terrenos, mantiene una relación en la que prácticamente no existen mediaciones; por tanto, no resultará sorprendente hallar que el extraordinario florecimiento de la nueva arquitectura posmoderna se basa en el mecenazgo por parte de los negocios multinacionales, cuya expansión y cuyo desarrollo son estrictamente contemporáneos con esta arquitectura.

Si bien no se trata de una obra ${ }^{12}$ estrictamente histórica, es interesante incluir en este resumen de posturas dirigidas al análisis de la modernidad aquél que toma como objeto de estudio al capitalismo, no sólo como una 
concepción económica o política en sí sino como un dispositivo que obra como readaptador de lo que lo critica, que es el sesgo principal de los estudios orientados por Luc Boltanski.

En efecto en tal voluminoso trabajo se intenta en primer término situar el estudio de la noción dentro de las variaciones históricas de un concepto amplio de ciudad, considerándose así seis tipologías en las que debe entenderse la función y acción que despliega ideológicamente la formación capitalista.

En la ciudad inspirada, la grandeza es la del santo que accede a un estado de gracia o la del artista que recibe la inspiración. Esta grandeza se revela en el propio cuerpo preparado mediante la áscesis y tiene en las manifestaciones inspiradas (santidad, creatividad, sentido artístico, autenticidad...) la forma de expresión privilegiada.

En la ciudad doméstica, la grandeza de la gente depende de su posición jerárquica en una cadena de dependencias personales. En una fórmula de subordinación establecida a partir de un modelo doméstico, el lazo político entre los seres es concebido como una generalización del lazo generacional que conjuga tradición y proximidad: el "grande» es el primogénito, el ancestro, el padre, a quien se debe respeto y fidelidad a cambio de protección y apoyo.

En la ciudad del renombre, la grandeza no depende más que de la opinión de los otros, es decir, del número de personas que otorguen su crédito y estima.

El "grande» en la ciudad cívica es el representante de un colectivo del que expresa la voluntad general.

En la ciudad comercial, el "grande» es aquel que se enriquece proponiendo sobre un mercado competitivo mercancías muy codiciadas, superando con éxito la prueba comercial.
En la ciudad industrial, la grandeza se funda en la eficacia y determina la configuración de una escala de capacidades profesionales.

Esta consideración es usada por estos autores como escena o pantalla socio-histórica en la cual o contra la cuál es preciso delinear la formulación nocional del capitalismo como ideología para luego plantear que en cierta forma el éxito o la pervivencia de tal ideología depende de su capacidad para adaptarse a las ciudades indicadas y también a un movimiento de deglución según el cual el capitalismo en un sentido inhabilita o desmonta sus críticas y en otro, las absorbe y operativiza.

Asi resulta interesante comprobar que los grandes sistemas críticos modernos -Boltanski y Chiapello hablan de la crítica artista y de la crítica social- se debaten en una productividad incapaz de abatir a su enemigo y además lo hacen malgré soi, aportándole ideas:

La crítica artista, aunque comparta con la modernidad su individualismo, se presenta como una contestación radical de los valores y opciones básicos del capitalismo: la crítica artista rechaza el desencanto resultante de los procesos de racionalización y de mercantilización del mundo inherentes al capitalismo, procesos que trata de interrumpir o suprimir, buscando de esa forma una salida al régimen del capital.

La crítica social, por su parte, trata de resolver ante todo el problema de las desigualdades y de la miseria, acabando con el juego de los intereses individuales. Aunque algunas de estas soluciones pueden parecer radicales no suponen, sin embargo, una paralización de la producción industrial, de la invención de nuevos artefactos, del enriquecimiento de la nación y del progreso material, constituyendo, por lo tanto, un rechazo menos total de los marcos y opciones del capitalismo. 
Sin embargo, a pesar de la inclinación predominante de cada una de estas dos críticas bien hacia la reforma, bien hacia la salida del régimen del capital, ambas poseen una vertiente moderna y una vertiente antimoderna. La tensión entre una crítica radical de la modernidad que conduce a «contestar su tiempo sin participar en él» y una crítica moderna que corre el riesgo de "participar en su tiempo sin contestarlo», constituye, de este modo, una constante de los movimientos críticos. La crítica artista es antimoderna cuando insiste en el desencanto y moderna cuando se preocupa por la liberación. Hundiendo sus raíces en los valores liberales provenientes del espíritu de la Ilustración, denuncia la falsedad de un orden que, lejos de llevar a cabo el proyecto de liberación de la modernidad, no hace sino traicionarlo: en lugar de liberar las potencialidades humanas de autonomía, de autoorganización y de creatividad, impide a la gente la dirección de sus propios asuntos, somete a los seres humanos a la dominación de las racionalidades instrumentales y les mantiene encerrados en una "jaula de hierro». La exigencia de la participación activa de los productores en el capitalismo no es sino la negación y destrucción de ésta.

La crítica social tiende a ser moderna cuando insiste en las desigualdades y antimoderna cuando, insistiendo en la ausencia de solidaridad, se construye como una crítica del individualismo.

Un tema que no podemos aquí más que apuntar es el de las relaciones entre lo moderno global universal o central y lo periférico, es decir, la instalación de la idea de modernidad en relación con los ambientes periféricos y la cuestión de las influencias, transculturaciones o traducciones. Allí emerge además la reversabilidad del camino de ida centro/periferia con sus tópicos de colonialidad y del camino de vuelta periferia/centro que suponen aprovechamien- tos globales de los aportes locales dentro de la óptica de la maximización general del mercado de la cultura y que empieza a poner en juego temáticas como la world music o la welt literatur.

Dentro de las cuestiones generales del poscolonialismo y el multiculturalismo destacan por ejemplo, las investigaciones del hindú Homi Bhabha ${ }^{13}$, quién en uno de sus más conocidos libros apunta lo siguiente:

Hay una conspiración de silencio alrededor de la verdad colonial, sea ésta cual sea. Silencio desde el imperio pero también silencio, mas ominoso, que profiere una arcaica otredad colonial, que habla en enigmas, obliterando los nombres propios y los lugares propios. Es un silencio que transforma el triunfalismo imperial, en el testimonio de la confusión colonial, y quienes oyen su eco pierden sus recuerdos históricos.

Es la voz de la literatura "colonial" del primer modernismo, la compleja memoria cultural de la que está hecha, en una excelente tensión entre desarraigo melancólico del novelista moderno, y la sabiduría tradicional, cuyo arte no trasciende a su propio pueblo. Un silencio repite siniestramente al otro.

El presente número 20 del regreso de Astrágalo, si bien no estrictamente monográfico, podría inscribirse en la temática de nuestro precedente ensayo centrado en la condición crepuscular (más que la auroral de la selva oscura del Dante, que entreveía la llegada del Renacimiento al final de ese medioevo que lo tuvo a tan mal traer) del ciclo de la modernidad y la condición de una posmodernidad que como bien la describe Antonio Fernández Alba (La quimera del fetiche fenicio) plantea en 
formas de arquitecturas de lo colosal y extravagante, la vuelta de aquellos objetos precisamente fetichizados y mercantilizados-fabricar el fetiche sin fisuras, entre lo económico y lo simbólico, dirá Fernández Alba- en diversas variantes de soliloquios enardecidos que se presentan despojados de la ética no consumada (aunque quizá consumida) de la modernidad dentro de la interesante noción de postrimerías icónicas, en que Fernández Alba alude lateralmente a ese motivo de las postrimerías tan propio del barroco fúnebre español.

Si la exasperación mercadotécnica que sucede a la posmodernidad (y bloquea la presunta libertad cultural-experimental de la posmodernidad) implica tecnificar de manera líquida la hasta ahora ontológica idea de naturaleza -conmovida por una forma productiva indiferente frente a su acelerada extinción- en el ensayo de Eduardo Grüner (La invención del desierto) se procede a la descripción de como un bioma natural deviene materia prima de acciones políticas y culturales para neutralizar dicha potencia natural, ya sea para concretar una invisibilización de quiénes habitaban dicho bioma (que entonces, no estaba desierto ni era inhumano), ya sea para convertirlo en referencia de cierta construcción estética (la asociación de soledad y autismo con la frugalidad estética minimalista) o espacio de mitificación de lo técnico-moderno capaz de apoderarse heroicamente del tal no-hábitat.

Otra mirada de final de modernidad es la que instituye la nueva idea-fuerza de memoria y patrimonio, como si se temiera que el festival de especulación financiera y el programa técnico de extinción completa de naturaleza también extinguiera los signos materiales -documentales y monumentales- del pasado. En el ensayo de Simón Marchan Fiz (Patrimonio: Resistir en la globalización) se argumenta sobre la mercantilización de aquellos signos mediante el peligro de acuñar una idea de patrimonio solamente relacionada con su valor de espectáculo, o sea, con montar políticas de patrimonio solo en cuanto este ítem devenga materia de rentabilidad.

La hipertrofia de tal noción de rentabilidad es lo que hace que Tom Angotti (Nueva York: ciudad compacta y sustentable?) se permita dudar de una especie de lugar común que suele vincular la capitalidad mundial de NY con calidades singulares de metropolineidad, ya que las políticas recientes no logran manhattanizar el área metropolitana ni hacerla eficiente o sustentable además de poner en evidencia condiciones de irracionalidad de tal área metropolitana en indicadores como la huella de carbono o el tiempo del conmuter casa-trabajo, revelando serias falencias de sustentabilidad. Una noción paradigmática de la calidad social de la modernidad - como fuera la creación de espacio público socialmente integrado- es revisada por Angotti como un serio déficit en los cuatro proyectos urbanos más ambiciosos del PLANYC2030 legado por el Alcalde Bloomberg bajo el incumplido lema $A$ Greener, Greater New York.

A caballo de leer un espacio metropolitano entre las trazas que le impone su desarrollosubdesarrollo y el registro de los sedimentos materiales de tal historia (que son menos trascendentes y/o valiosos que lo que describe la noción más amplia posible de patrimonio urbano-arquitectónico) el ensayo de Juan Bautista Ritvo (Frontera el despojo y fascinación por lo lejano) escrito en relación a la investigación-muestra Atlas/Archivo trata de elaborar, casi alrededor de expresiones sintomáticas cercanas a su mirada de analista, una reflexión sobre lo que queda útil o inútil, materia o residuo, testimonio o recuerdo, 
de la relación entre una sociedad urbana (Buenos Aires) con el soporte natural en que se instaló (la costa del Rio de la Plata) y que va más allá de la historia social por una parte y del análisis urbano por otra. El trabajo analizado a su vez, trae a colación la posibilidad de utilizar el método warburgiano de la mnemosyne para expandirlo de su inicial utilización estético-historiográfica hasta un modo de coleccionar-representar la multiplicidad a veces incongruente de los innumerables registros de la historia urbana y territorial (desde postales y fotos familiares, fragmentos periodísticos y estudios socio-naturales hasta guías turísticas y cartografías científicas).

El ensayo de Antonio Toca Fernández (El tejido de la arquitectura) se centra en rescatar de aquel legado de modernidad susceptible de confrontar la evanescencia puramente sígnica de lo posmoderno, la idea de tectónica que recientemente reinstaló de manera crítica Frampton, revisando la complejidad de las teorías estéticas semperianas acerca de la relación entre constructo tectónico y envolvente urdida o tejida y de allí valorar la idea moderna de una arquitectura de pieles asociadas al savoir-faire del tejedor. Lo moderno esponjará la tectónica lítica clásica y usará la poética industrial para tejer sus redes.

El esponjamiento moderno, capaz de entender a lo urbano como el espacio entre las cosas es lo que proponen en su ensayo Pedro Livni Gonzalo-Carrasco Purull (La ciudad, el espacio entre las cosas) a partir de una sugestiva interpretación del plus que Gordon Cullen agregó, en plan fenomenologista, a su capacidad de observación/representación haciendo que una serie final de sus dibujos adquiriera la dimensión de una lectura analítica de lo urbano a partir del doméstico espacio en que estaba dibujando.
Lo atmosférico pero en tanto variante extrema de la desmaterialidad asociada a la racionalidad energética es lo que propone en su estudio Eduardo Prieto (Eclecticismo Atmosféri$c o$ ), fragmento de su tesis doctoral madrileña que indaga en las nuevas relaciones entre estéticas y crisis de sustentabilidad, alrededor de diseñadores recientes más interesados en sus procesos de análisis y crítica que en la oferta de nuevos objetos.

Fernando Diez en cambio propone una investigación más clásico-moderna y de sabor a lo Colin Rowe (Espacios subordinantes y subordinados en el proyecto contemporáneo) al conjuntar si se quiere las miradas de Fernández Toca sobre la piel tectónica, la de Livni-Carrasco sobre el espacio-entre-las-cosas y la de Prieto sobre las últimas experiencias de desmaterialidad, al volverse a plantear una revisión de la célebre registración de lleno-vacío de Nolli para indagar en nuevas variantes de la relación entre continente y contenido, entre estructura y espacio.

El proceso moderno de transformación territorial uno de cuyos episodios fallidos debido a los excesos capitalistas (ese modo productivo capaz de convertir su propio proceso de destrucción de la naturaleza en espectáculo según lo apuntara Eduardo Subirats) obliga a tratar de entender eso que solemos llamar infraestructuras, lo que coloca al ensayo de Diego Capandeguy-Thomas Spreechman-Federico Gastambide (Sobre las infraestructuras y otros ordenes territoriales) en el camino de intentar pensar esa mega-transformación no en una fatalidad técnico-económica sino en una necesaria nueva de escala de proyecto a ser pensada precisamente desde el proyecto.

En la sección que inauguramos -Espacio de Libros de Espacio- nos proponemos presen- 
tar la argumentación crítica de libros recientes que ayuden a entender las complejidades propias de la crisis de la modernidad en su relación con la cultura de las ciudades. Proyectos y Aparatos revisa la noción de aparato -como la perspectiva o la fotografía- propuesta por Jean Louis Deotte indagando si además de describir conceptualmente fenómenos de modernidad pudiera ser convergente a una redefinición de la noción de proyecto. Lechos inhóspitos revisa el apocalíptico libro de Johnatan Crary referido a la ampliación total del tiempo de producción, tendiendo a que el descanso ocioso o el sueño sean también oportunidades de maximizar el capital. Escrituras de Luz es la recensión de la investigación de Eduardo Cadava que a partir de las famosas tesis históricas de Benjamin y otros de sus textos, se inserta en el creciente interés de las imágenes por sobre los textos y en la posibilidad de fundar una textualidad basada en rasgos de luz. Y Ciudad del Arte alude a un libro del curador y teórico de arte alemán Boris Groys que en la línea de las ideas de Beuys -todos somos artistas- analiza la expansión del arte en sujetos que lo producen y consumen y en medios que lo multiplican y quizá también lo pongan en crisis, como Internet.

\section{NOTAS}

1 Veáse mi libro Utopías Sociales y Cultura Técnica. Estudios de Historia de la Arquitectura Moderna, Concentra, Buenos Aires, 2005, que desarrolla temas que van de 1820 a 1940.

2 Aludo aquí a Espacio. Tiempo y Arquitectura y a La Mecanización toma el mando, respectivamente editadas en 1941 y en 1946, el primero en Europa y el segundo en USA.

3 Colominas, B., Doble exposición. Arquitectura a través del arte, Akal, Madrid, 2006. El ensayo que estudia el rol de LC en relación al arte de vanguardias es el 2, Le Corbusier y Duchamp: el inseguro estatus del objeto, página 35.

4 Burger, P., Teoría de la Vanguardia, Editorial Península, Barcelona, 1987.

5 Bourriaud, N., Post-producción. La cultura como escenario: modos en que el arte reprograma el mundo contemporáneo, Editorial A. Hidalgo, Buenos Aires, 2004. Lo que realmente importa-dice Bourriaud-es lo que hacemos con los elementos puestos a nuestra disposición. Somo entonces locatarios de la cultura; la sociedad es un texto cuya regla lexical es la producción, una ley que corroen desde adentro los usuarios supuestamente pasivos a través de las prácticas de postproducción. Cada obra -cita Bourriaud a De Certeau-es habitable a la manera de un departamento alquilado (p.23).
6 Tafuri. M., Teoría e Historia de la Arquitectura, Celeste, Madrid, 1997. En el capítulo denominado La Arquitectura Moderna y el Eclipse de la Historia de dicho libro Tafuri esboza un criterio para situar un posible origen de la modernidad arquitectónica al menos referido al caso italiano pero consciente de cómo el mismo contribuye a la fundación disciplinar moderna: Desde el momento en que Brunelleschi institucionaliza un código lingüístico y un sistema simbólico basándose en la comparación suprahistórica con el gran ejemplo de la antigüedad, hasta el momento en que Alberti no se contenta y a con un historicismo mítico, y explota racionalmente la estructura de aquel código en sus valores sintácticos y en sus valores emblemáticos, en este lapso de tiempo, decíamos, se quema el primer gran intento de la historia moderna de actualización de los valores históricos como traducción de un tiempo mítico a un tiempo presente, de significados arcaicos a mensajes revolucionarios, de 'palabras' antiguas a acciones civiles"...

7 Toulmin, S., Cosmópolis, Península, Barcelona, 2001. En especial la discusión presentada en el Capítulo 1, Qué problema plantea la modernidad?, que es donde se plantea la peculiar modernidad corta de la arquitectura -iniciada por sus exegetas historiográficos hacia 1890- que además posee la curiosa enunciación de un fin o cese de la misma (planteado según Toulmin, por Robert Venturi hacia los 70) junto 
al inicio de otra fase histórica llamada postmodernidad que Toulmin prefiere adjudicar como innovación historiográfica más que a artistas y arquitectos a críticos sociales que como Peter Drucker indicaban hacia 1957 un fin de una idea de modernidad que habiendo comenzado hacia el siglo XVII estaba para entonces extinguiéndose básicamente debido al inicio de la caducidad de la idea de Estado-Nación.

8 Sloterdijk, P. Derrida, un egipcio. El problema de la pirámide judía, Amorrortu , Buenos Aires, 2007, ensayo 4, Franz Borkenau y Derrida.

9 Habermas, J., El discurso filosófico de la modernidad, Taurus, Madrid, 1989.
10 Jauss, H.R., Las transformaciones de lo moderno. Estudios sobre las etapas de la modernidad estética, La Balsa de la Medusa, Madrid, 1995.

11 Jameson, F., Ensayos sobre el Posmodernismo, Editorial Imago Mundi, Buenos Aires, 1991, p.20.

12 Boltanski, L.- Chiapello,E. El nuevo espiritu del capitalismo, Editorial Akal, Madrid 2002.

13 Bhabha, H., The Location of Culture, Routledge, Londres, 1998, cita proveniente del capítulo VII, Articular lo arcaico: Diferencia cultural y sinsentido colonial. 


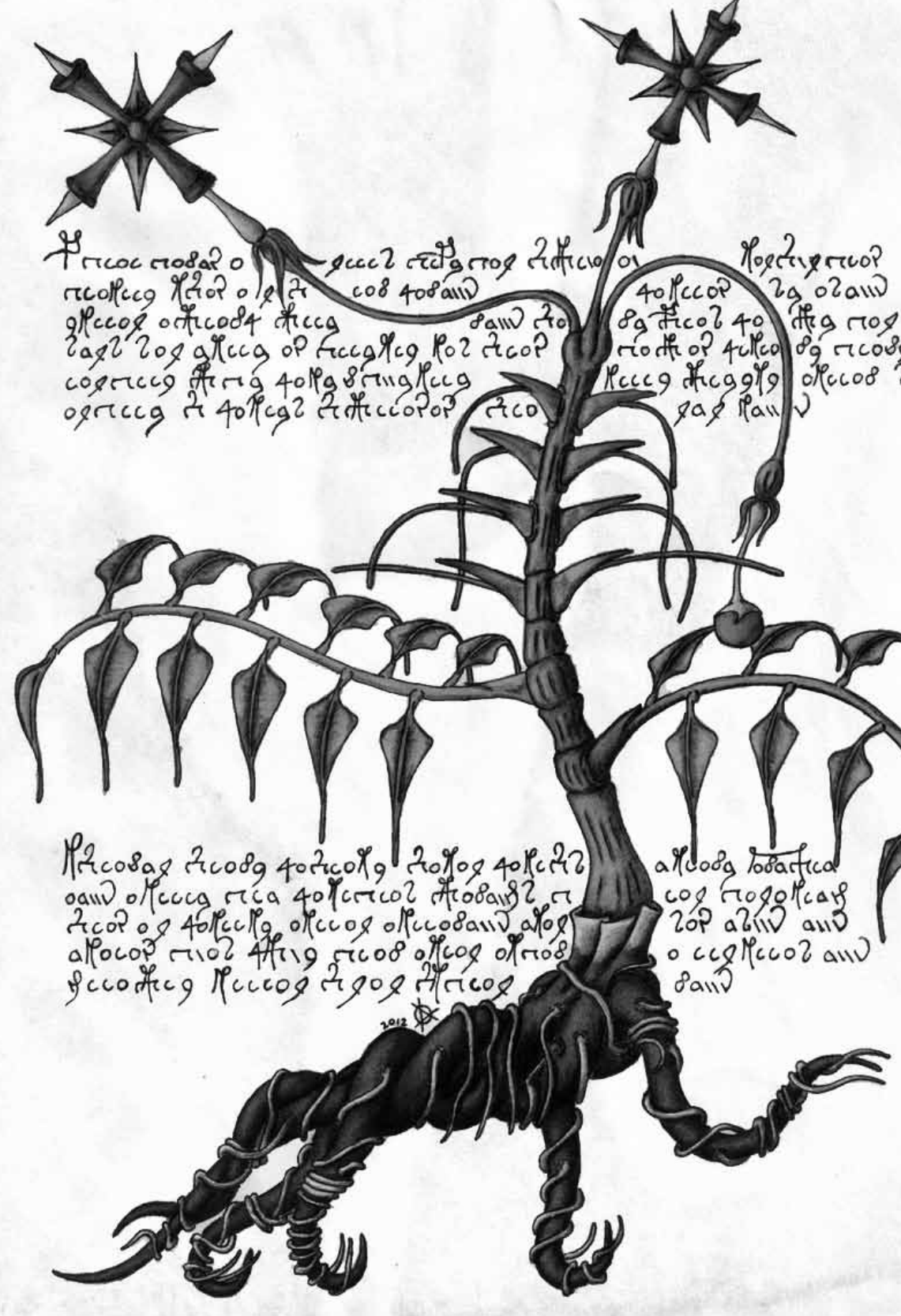

